\title{
Die KRGÖ im Jahr 2016
}

Im Berichtsjahr konnten die Kommission für Rechtsgeschichte der ÖAW (KRGÖ-AW) sowie die Abteilung KRGÖ an der Universität Wien (KRGÖ-UW) - zusätzlich zum regelmäßigen Erscheinen der gegenständlichen Zeitschrift insgesamt vier Bücher herausbringen:

In der Reihe „Fontes iuris" erschien als Band 24 eine Edition von Instruktionen und anderen normativen Quellen aus dem 17., 18. und 19. Jh. zur Verwaltung der liechtensteinischen Herrschaften Feldsberg [Valtice/CZ] und Wilfersdorf in Niederösterreich (Anita HIPFINGER [Hg.], „Das Beispiel der Obrigkeit ist der Spiegel des Unterthans“, Wien-Köln-Weimar 2016). Dieser Band ist Teilergebnis eines von Kommissionsmitglied Thomas WiNKELBAUER am Institut für Geschichte der Universität Wien durchgeführten FWF-Projekts zur Herrschaftsverwaltung in Niederösterreich (Projekt P 20350); ein zweiter Band aus diesem Projekt, enthaltend Quellen zur Grundherrschaft Klosterneuburg, soll in Bälde in den „Fontes iuris“ erscheinen.

Aus dem von Kommissionsobmann OleCHOWSKI geleiteten und teils an der ÖAW, teils an der Universität Wien durchgeführten FWF-Projekt „Hans Kelsens Leben in Amerika“ (Projekt P 23747) gingen 2016 zwei Buchpublikationen hervor: Beim ersten handelt es sich um ein von einem vormaligen KRGÖ-Mitarbeiter erstelltes deutsch-englisches Glossar zu Kelsens Rechtslehre und Demokratielehre (Ramon PILS, Terminologie Hans Kelsen, Schriftenreihe des Hans Kelsen-Instituts 37, Wien 2016). Der Sammelband "Das internationale Wirken Hans Kelsens" (hg. v. Clemens JABLONER, Thomas OLECHOWSKI und Klaus ZELENY, Schriftenreihe des Hans Kel-
sen-Instituts 38, Wien 2016) enthält zehn Beiträge von OLECHOWSKI, der beiden vormaligen KRGÖ-Mitarbeiter Miriam GASSNER und Stefan WEDRAC sowie von weiteren Wissenschaftern aus Brasilien, Italien, Liechtenstein, Schweden, Tschechien, Zypern und Österreich, die die Tätigkeit des großen Juristen als Gutachter für Regierungen, seine Vortragsreisen nach Skandinavien, die Resonanz seines Schaffens in der Sowjetunion und andere Aspekte seines globalen Wirkens beleuchteten.

BRGÖ-Chefredakteur Christoph SCHMETTERER, der auch am vorhin genannten Band 38 mitwirkte, veröffentlichte 2016 - aus Anlass des 100. Todestages von Kaiser Franz Joseph - eine Biographie dieses Monarchen (,Kaiser Franz Joseph I.“, Wien-Köln-Weimar 2016), die nicht nur, aber auch dessen Bedeutung für die österreichische Rechtsgeschichte unterstreicht.

Auch im Rahmen des Langzeitprojekts „Erschließung der Akten des kaiserlichen Reichshofrates" der Akademie der Wissenschaften zu Göttingen konnte, in Kooperation mit der KRGÖ-UW, ein weiterer Erschließungsband herausgegeben werden („Serie II: Antiqua, Band 3“, hg. v. Wolfgang SELLERT, Berlin 2016).

Die Kommissionsmitglieder KALB, OleCHOWSKI und ZIEGERHOFER erstellten gemeinsam mit Laura RATHMANNER (KRGÖ an der Universität Wien) und dem vorhin genannten Stefan WEDRAC (mittlerweile am Institut für Neuzeitund Zeitgeschichtsforschung der ÖAW tätig) einen Antrag für ein neues Projekt, das der FWF in seiner Sitzung vom 28. November 2016 genehmigte: „Die rechtliche Bedeutung des Vertrags von St. Germain“ (FWF-Projekt P 29774). 
Im Rahmen des Projekts soll der erste umfassende Kommentar zu jenem Vertrag erstellt werden, den die junge Republik Österreich nach dem Ersten Weltkrieg, am 10. September 1919, mit den Siegermächten unterzeichnete. Er soll rechtzeitig zum 100-Jahr-Jubiläum dieses Vertrages 2019 fertiggestellt werden.

Im Sommer 2016 unterzog das Rektorat der Universität Wien die KRGÖ-UW einer Evaluation. Aufgrund der positiven Ergebnisse derselben beschloss das Rektorat am 13. September, die KRGÖ-UW in der bisherigen Form, aber unter dem neuen Namen "Forschungsstelle für Rechtsquellenerschließung" (FRQ) weiterzuführen. Der neue Name soll nicht zuletzt zu einer besseren Unterscheidung von der KRGÖ-AW beitragen, mit der die neue Forschungsstelle gleichwohl auch künftig eng zusammenarbeiten wird. Die Leitung der Forschungsstelle obliegt dem derzeitigen Kommissionsobmann, wobei hier betont sein soll, dass er all diese Tätigkeiten, wie stets, unentgeltlich vollbringt.

Was die Mitarbeiter/innen der KRGÖ-UW (künftig: FRQ) betrifft, so ist hier anzuführen, dass Frau Mag. Laura RATHMANNER, bislang freie Dienstnehmerin, mit 1. März 2016 eine Anstellung als Universitätsassistentin erlangte. Frau Univ.-Ass. Mag. Kamila Maria STAUdIGLCIECHOWICZ LL.M. unterbrach in den Monaten Oktober und November 2016 ihre seit Dezember 2015 dauernde Elternkarenz, in die sie im An- schluss zurückkehrte. Neu hinzu kam Frau Melina VERYKIOS als freie Dienstnehmerin. Der freie Dienstvertrag mit Herrn Univ.-Lektor RA DDr. Christoph SCHMETTERER wurde weiter verlängert. Frau Katharina BERNOLD befand sich das ganze Jahr 2016 über in Elternkarenz.

Die KRGÖ-UW verbrauchte im Berichtszeitraum aus dem Budget der Universität Wien Personal- und Sachmittel in Höhe von $€$ 33.268,87; die finanziellen Zuwendungen der ÖAW an die KRGÖ-AW betrugen im selben Zeitraum $€$ 3.700,- (einschließlich einer finanziellen Förderung des Lektorats der gegenständlichen Zeitschrift). Für die genannten, aus FWFProjekten hervorgegangenen Publikationen gewährte der FWF einen Druckkostenzuschuss von insgesamt $€ 27.618$,- (womit auch die open access-Kosten abgedeckt wurden). Für die im Dezember 2015 stattgefundene Tagung „Gerichtsvielfalt in Wien" und den dazugehörenden Tagungsband (= BRGÖ 2016/2) wurden im Berichtszeitraum weitere Drittmittel aus den Förderungen der Stadt Wien in Höhe von $€ 1.200$,aufgebraucht. Im Rahmen des ReichshofratsProjekts kamen $€ 35.664,36$ von der Akademie der Wissenschaften zu Göttingen hinzu. Das Gesamtbudget beider Forschungseinheiten setzte sich somit zu $32 \%$ aus Mitteln der Universität Wien, zu $5 \%$ aus Mitteln der ÖAW und zu $63 \%$ aus Drittmitteln zusammen.

Thomas OLECHOWSKI 